\title{
Étude de la stabilité de quelques propriétés physico- chimiques des tranches d'igname congelées (Dioscorea cayenensis-rotundata cv Kponan) de Côte d'Ivoire et analyse sensorielle des mets dérivés
}

\author{
Coulibaly Aïssatou ${ }^{1 *}$; Kouadio Degbeu Claver; Doh Amenan Aline; Amani N'Guessan Georges ${ }^{1}$ \\ 1 Université Nangui Abrogoua, Abidjan, Côte d'Ivoire 02 BP 801 Abidjan 02 \\ Auteur correspondant : aiscool@yahoo.fr
}

Original submitted in on 22nd July 2020. Published online at www.m.elewa.org/journals/ on $28^{\text {th }}$ February 2021

https://doi.org/10.35759/JABs.158.6

\begin{abstract}
RÉSUMÉ
Objectif : Évaluer l'impact de la congélation sur certains paramètres physico-chimiques des tranches d'ignames de la variété kponan et faire une analyse sensorielle de quelques mets dérivés.

Méthodologie et résultats : L'étude a été faite sur des tranches d'ignames prétraitées puis congelées pendant 3 mois. À Chaque mois, des échantillons ont été prélevés pour une analyse des paramètres physicochimiques et sensorielle des mets dérivés. La congélation n'a pas eu d'effet sur les teneurs en fibre, en protéine et en cendre. Cependant, au cours du premier mois, la matière sèche a augmenté de 5,59\%. Une augmentation des sucres réducteurs a été également observée. Tandis que la teneur en amidon a diminué de $5,54 \%$. A partir du deuxième mois, tous les paramètres physico-chimiques étudiés sont demeurés stables. Le profil sensoriel des mets dérivés a montré une bonne appréciation des caractéristiques sensorielles avec une forte élasticité (foutou) et une augmentation de la croustillance, de la fermeté et de la couleur (frite).

Conclusion et applications des résultats : La technologie utilisée au cours de cette étude a permis de conserver la majeure partie des caractéristiques physico-chimiques et sensorielles de l'igname durant 3 mois. La production de tranches d'igname congelées doit être envisagée afin de palier le problème de conservation post-récolte, assurer la disponibilité tout au long de l'année permettant d'assurer la sécurité alimentaire et contribuer ainsi à la lutte contre la pauvreté en milieu rurale.
\end{abstract}


Coulibaly et al., J. Appl. Biosci. 2021 Étude de la stabilité de quelques propriétés physico-chimiques des tranches d'igname congelées (Dioscorea cayenensis-rotundata cv Kponan) de Côte d'Ivoire et analyse sensorielle des mets dérivés

Study of the stability of some physicochemical properties of frozen yam slices (Dioscorea cayenensis-rotundata CV Kponan) from Côte d'Ivoire and sensory analysis of derived dishes

\begin{abstract}
Objective: Evaluate the impact of freezing on certain physicochemical parameters of yam slices of the kponan variety and make a sensory analysis of some derived dishes.

Methodology and results: The study was performed on slices of pretreated yams and then frozen for 3 months. Each month, samples were taken for an analysis of the physico-chemical and sensory parameters of the derivative dishes. Freezing did not affect fiber, protein, and ash contents. However, in the first month, dry matter increased by $5.59 \%$. An increase in reducing sugars was also observed. While the starch content decreased by $5.54 \%$. From the second month, all the physico-chemical parameters studied remained stable. The sensory profile of the derived dishes showed a good appreciation of the sensory characteristics with high elasticity (foutou) and an increase in crispness, firmness, and color (fried).

Conclusion and application of the results: The technology used during this study made it possible to conserve most of the physico-chemical and sensory characteristics of the yam for 3 months. The production of frozen yam slices should be considered in order to overcome the post-harvest conservation problem, ensure availability throughout the year, ensuring food security and thus contributing to the fight against poverty in the environment. rural.
\end{abstract}

\section{INTRODUCTION}

La culture de l'igname contribue à la sécurité alimentaire de plus de 300 millions de personnes à travers le monde (FAO, 2009 ; Kouakou, Zohouri, Dibi, N'Zué, \& Foua-Bi, 2012). L'igname est une importante source alimentaire de première importance pour les populations d'Asie, des Caraïbes, du pacifique, d'Amérique et d'Afrique subsaharienne (Bakayoko, Kouamé, \& Boraud, 2017 ; International Institute of Tropical Agriculture (IITA), 2020). En Côte d'Ivoire, l'igname occupe le premier rang parmi les cultures vivrières non céréalières avec une production supérieure à 7 millions de tonnes sur une superficie de plus de 990 000 ha (FAOSTAT, 2018). Les tubercules sont constitués en majorité d'hydrate de carbone qui représente $90 \%$ de la matière sèche dont l'élément principal est l'amidon. Outre les glucides, ils renferment aussi des protéines, des lipides, des vitamines et des sels minéraux en faible quantité mais non négligeable (Konan, Bédié, \& Borda, 2015). Sa consommation annuelle en Afrique de I'Ouest est estimée à 61 kilogrammes par habitant sous forme de mets tels que la bouillie, le foutou, le foufou et les frites. En Afrique, ils sont également écrasés en pâte collante ou en pâte après l'ébullition (International Institute of Tropical Agriculture (IITA), 2020). En plus de son importance nutritionnelle, l'igname occupe également une fonction économique. En effet, sa commercialisation représente une source de revenu pour les acteurs du domaine (producteurs, transformateurs et commerçants (Kouame et al., 2016). Selon Asiedu (2003), elle constituerait une source financière importante pour les femmes majoritairement impliquées dans la production, la transformation et la commercialisation. Malgré son importance nutritionnelle et économique, la conservation post récolte de l'igname constitue un véritable problème pour les acteurs. Les pertes post-récoltes sont assez importantes et estimées de 25 à $65 \%$ (FIRCA, 2010) en dépit des techniques traditionnelles existantes de conservation et du phénomène de dormance. Ces derniers ont montré des limites quant à l'action des ravageurs, des maladies, des nématodes ainsi que les dégâts mécaniques et la germination. Toutes ces menaces entrainent le pourrissement, la réduction de la qualité et les possibilités de commercialisation et ainsi une baisse de revenus pour les acteurs. Afin de pallier ces insuffisances, de plus en plus d'études sont menées sur les aliments congelés voire surgelés en raison de l'accroissement du désir de commodité en termes de temps et d'énergie nécessaires à la préparation finale de la nourriture 
pour le consommateur (Saxena, Raju, \& Bawa, 2014). De plus, la conservation au froid présente de nombreux avantages. En effet, la congélation est une méthode rapide, pratique et populaire de conservation des aliments. Si la congélation est faite correctement, elle conserve les aliments sans entrainer des changements au niveau de la taille, de la forme, de la texture, de l'arôme et des nutriments. Cette technologie a déjà montré son efficacité dans le domaine industriel et est utilisée pour la

\section{MATÉRIEL ET MÉTHODES}

Source du matériel végétal : Le matériel biologique utilisé pour la réalisation de cette étude est l'igname de la variété Kponan de l'espèce Dioscorea cayenensisrotundata (figure 1). Les tubercules d'igname ont été achetés au marché local d'Abobo à Abidjan en Côte d'Ivoire chez des grossistes et acheminés au conservation des fruits, légumes, viandes, poissons et laits (Sun, 2012). Une méthode de conservation sur une longue période serait un catalyseur du développement rural entrainant une augmentation des revenus des producteurs, des transformateurs et des commerçants. L'objectif de ce travail était d'étudier l'aptitude des tranches d'ignames à la congélation sans variation des constituants biochimiques et de faire une analyse sensorielle des mets dérivés.

Laboratoire de Biochimie Alimentaire et Technologies des Produits Tropicaux (LBATPT) de l'Université Nangui Abrogoua pour les différents tests. Les équipements et les réactifs utilisés pour les expériences sont de norme et de qualité standards.

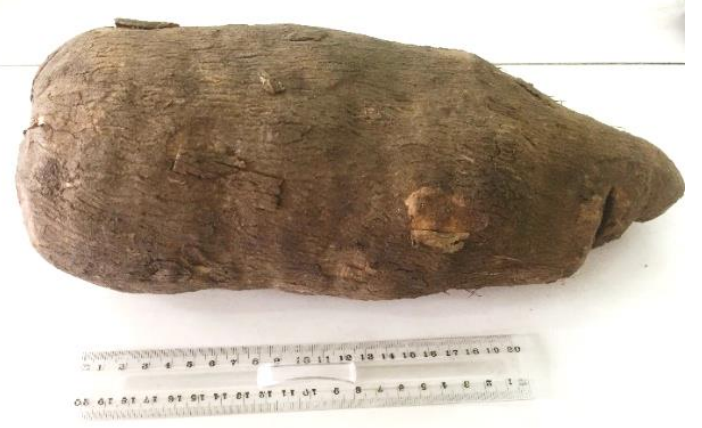

Figure 1: Tubercule d'igname

Préparation des échantillons: Les tubercules d'ignames ont été soigneusement lavés à l'eau de robinet, pelés puis découpés à l'aide d'un couteau en acier inoxydable en tranche de $50 \mathrm{~g}$ de longueur $6 \times 4$ $\mathrm{cm}$ pour les tranches qui ont servi à la préparation de l'igname bouillie et du foutou igname. Celles destinées à la friture étaient des tranches de $30 \mathrm{~g}$ de longueur $10 \times$ $6 \mathrm{~cm}$. Les tranches d'ignames ont été prétraitées selon la méthode décrite par Coulibaly et al (2019). Elles ont été trempées pendant 30 minutes dans du jus de citron à $\mathrm{pH} 3$, utilisé comme agent anti-brunissement naturel puis blanchies à $85^{\circ} \mathrm{C}$ pendant 2 minutes. Les tranches prétraitées ont été divisées en quatre lots puis conditionnés dans des sacs de congélation en polyéthylène tout en veillant à ce que les sacs soient dépourvus d'air. Trois de ces lots ont été conservés au congélateur à $-18^{\circ} \mathrm{C}$ et des prélèvements périodiques ont été effectués sur ces derniers à chaque mois sur une période de 3 mois. Le dernier lot n'ayant pas été congelé a servi de témoin. Les échantillons obtenus ont été notés ITC0, ITC1, ITC2, ITC3 correspondant respectivement aux ignames traitées et non congelées, ignames traitées et congelées à 1 mois, ignames traitées et congelées à 2 mois et ignames traitées et congelées à 3 mois (figure 2). 


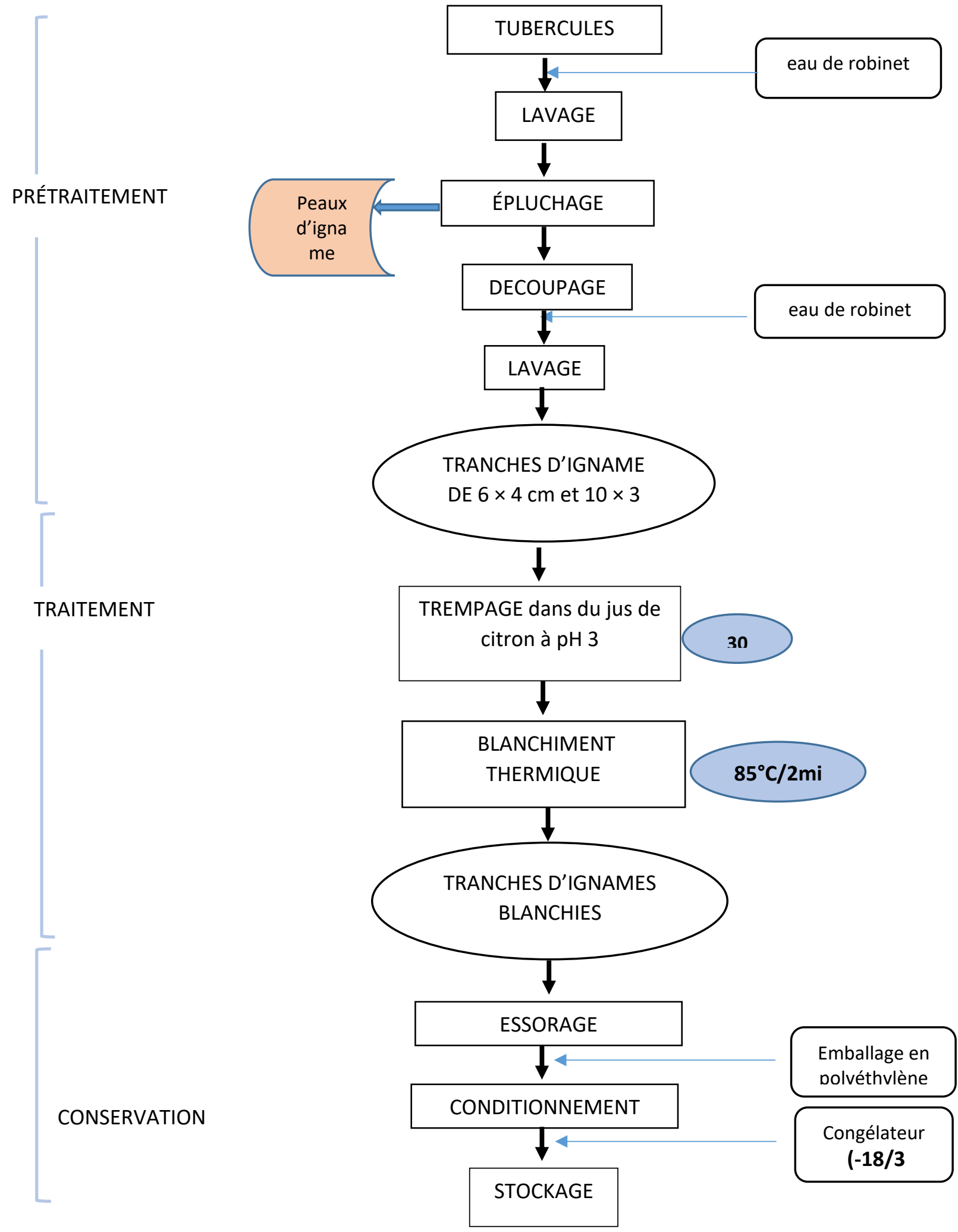

Figure 2: Diagramme de production des tranches d'igname congelées 
Méthode d'analyse

Détermination de la matière sèche: Le taux de matière sèche des différentes tranches d'ignames a été déterminé selon la méthode AOAC (1990). Cinq grammes $(5 \mathrm{~g})$ d'échantillon d'igname prélevé après la congélation ont été pesés $\left(P_{1}\right)$ à l'aide d'une balance (SARTORUIS BP 310S, Allemagne) dans un creuset de masse connue $\left(P_{0}\right)$. Ils ont été séchés dans une étuve (BIOBASE, Model : BOV-V125F), à $100 \pm 5^{\circ} \mathrm{C}$ pendant $24 \mathrm{~h} \pm 30 \mathrm{mn}$ jusqu'à obtenir une masse constante. Les échantillons ont été refroidis dans un dessiccateur dès la sortie de l'étuve et pesés $\left(P_{2}\right)$. La teneur en matière sèche a été déterminée à partir de l'équation suivante :

$$
\operatorname{TMS}(\%)=\frac{\left(\mathbf{P}_{2}-\mathbf{P}_{0}\right)}{\mathbf{P 1}} \times 100
$$

Extraction d'amidon: L'extraction de l'amidon s'est faite selon la méthode modifiée décrite par Amani et al. (2004). Les tubercules ont été lavés, épluchés et découpés en petits morceaux. Les petits morceaux ont été séchés à l'étuve à $55^{\circ} \mathrm{C}$, puis broyés dans un mixeur de marque Moulinex (France). La poudre obtenue est reprise dans une solution de chlorure de sodium (4\% $P / V)$ afin de séparer les protéines de l'amidon puis passée à travers une série de tamis dont les mailles sont respectivement 500, 250, $100 \mu \mathrm{m}$ de diamètre. Le lait d'amidon obtenu a subi une alternance de décantation et de lavage (4 fois au moins). Le produit obtenu a été mis à sécher à l'étuve à $45 \mathrm{C}$ pendant 48 heures.

Dosage des sucres réducteurs: La méthode de Bernfeld (1955) utilisant l'acide 3,5-dinitrosalicylique (DNS) a été utilisée pour le dosage des sucres réducteurs . Dans cette méthode, la teneur en sucres réducteurs de $0,5 \mathrm{~g}$ d'échantillon est déterminée après extraction et purification, sur un courbe étalon tracé en fonction de la concentration et de la densité optique de solutions de glucose de concentrations connues. Des solutions de sucres de concentrations inconnues ont été déterminées à l'aide d'un spectromètre à des densités optiques de $546 \mathrm{~nm}$. La concentration en sucres réducteurs des échantillons est déterminée grâce aux densités optiques rapportées sur la courbe étalon..

Détermination de la teneur en Protéines : La méthode de Lowry et al. (1951) a été utilisée pour la détermination des teneurs en protéines des solutions enzymatiques. . Lorsque les protéines sonten contacte avec le réactif de Folin-Ciocalteu (constitué de tungstate et de molybdate de sodium en solution dans l'acide phosphorique et l'acide chlorhydrique), elles forment des complexes colorésqui résultent de la réaction du phosphomolybdate par la tyrosine et le tryptophane. L'intensité de la coloration est fonction de la teneur en acides aminés aromatiques présents ainsi que des protéines. La lecture des densités optiques est faite à $660 \mathrm{~nm}$ contre un témoin contenant tous les réactifs excepté les protéines. Cette méthode permet le dosage des concentrations de protéine allant de 5 à $100 \mu \mathrm{g} \cdot \mathrm{ml}^{-1}$

Dosage de fibres totaux : Pour connaître la teneur en fibres totaux, on porte à ébullition $1 \mathrm{~g}$ d'échantillon (M) dans $50 \mathrm{ml}$ d'acide sulfurique $(0,25 \mathrm{~N})$ et ensuite dans $50 \mathrm{ml}$ de soude $(0,31 \mathrm{~N})$ pendant une heure (30 minutes $x 2$ ). on sèche à $105^{\circ} \mathrm{C}$ pendant 8 heures (M1) le résidu obtenu puis on l'incinère à $550^{\circ} \mathrm{C}$ pendant 3 heures (M2). La teneur en fibres brutes (F B) est exprimée selon par la formule ci-dessous :

$$
\text { FB }(\%)=\frac{(M 1-M 2)}{(M \times M S)} \times \frac{100}{M S(\%)}
$$


Détermination du taux de cendres : Les cendres ont été déterminées selon la méthode de l'AOAC (1990). Dans un creuset préalablement taré on a mis un gramme de chaque échantillon d'igname. La masse d creuset et échantillon est notée Mce. L'ensemble est placé dans un four à moufle à $550^{\circ} \mathrm{C}$ pendant 48 heures. Après incinération, l'ensemble Mcc est retiré du four et déposé dans un dessiccateur pour refroidissement. On effectue ensuite les pesées. Le taux de cendre est déterminé par la formule suivante :

$$
\% \text { Cendre }=\frac{(\text { Mcc }- \text { Mcv })}{(\text { Mce }- \text { Mcv })} \times 100
$$

Analyse sensorielle: Les préparations culinaires retenues au cours de cette étude étaient l'igname bouillie, le foutou d'igname et l'igname frite. Les tests descriptifs ont permis de mettre en évidence l'impact de la congélation sur les différents paramètres organoleptiques des mets dérivés de tranches d'ignames congelées lors des différents mois de conservation. La détermination des caractéristiques organoleptiques a été faite par 15 dégustateursconsommateurs habituels des mets. L'échelle de notation était une fiche d'évaluation élaborée pour tenir compte de la spécificité des ignames bouillies, du foutou igname et des ignames frites. Les caractéristiques retenues du foutou d'igname étaient la couleur, l'apparence, l'élasticité (avec la fourchette, les doigts et dans la bouche), l'adhésivité, le goût et l'odeur. L'igname bouillie était caractérisée par la couleur, la fibrosité, la fermeté (avec la fourchette, les doigts et dans la bouche), le goût et l'odeur. En fin la couleur, la fermeté (avec la fourchette, les doigts et dans la bouche), la croustillance (avec les doigts et dans la bouche), le goût

\section{RÉSULTATS}

Évolution des paramètres physico-chimiques au cours de la conservation: Le Tableau 1montre l'évolution de certains paramètres physico-chimique au cours des 3 mois de congélation des tranches d'ignames. Les résultats ont montré qu'il n'existe aucune évolution significative $(P \geq 0,05)$ de la teneur en fibre, protéine et cendre au cours des 3 mois de congélation. Contrairement à eux, la matière sèche, l'amidon et les sucres réducteurs évoluent significativement $(P<0,05)$. et l'odeur ont été retenus pour l'évaluation des frites d'ignames. Les notes attribuées étaient comprises entre 1 et 5 et correspondent aux appréciations suivantes : 1 = pas $; 2=$ peu $; 3=$ moyen $; 4=$ très $; 5=$ extrêmement. Les tests se sont déroulés dans une salle bien aérée à l'abri de toutes odeurs (LBATPT) ainsi que des distractions auditives et visuelles. Les échantillons ont été codés avec des numéros à 3 chiffres et présentés aux dégustateurs dans un ordre choisi au hasard sous la forme de mets d'igname bouillie, de foutou d'igname et d'igname frite ordinaire. Les dégustateurs ont bu de l'eau entre la dégustation des différents échantillons préparés et n'ont pas communiqué entre eux pendant la durée de l'évaluation.

Analyse statistique des résultats: Des analyses de variances (ANOVA) ont été effectuées sur les résultats avec le logiciel Stastica 7.1 en vue de comparer les moyennes. Lorsqu'il y avait une différence significative, le test de Tukey a permis d'identifier les moyennes impliquées dans la différence observée au seuil de $5 \%$.

La teneur en matière sèche augmente de $5,59 \%$ après 1 mois de congélation et reste constante durant les 2 derniers mois. La teneur en sucre réducteur augmente de $6,02 \pm 0,13$ à $12,65 \pm 0,42 \mathrm{~g} / 100 \mathrm{~g} \mathrm{MS}$ ) après 1 mois de congélation et se stabilise aussi les 2 derniers mois. La teneur en amidon baisse significativement de 5,54\% après 1 mois de congélation. Les 2 derniers mois, la teneur en amidon demeure stable. 
Tableau 1 : Évolution de la composition biochimique de l'igname Kponan au cours de la congélation.

\begin{tabular}{|c|c|c|c|c|}
\hline $\begin{array}{l}\text { Paramètres } \\
\text { Biochimiques }\end{array}$ & ITCO & ITC1 & ITC2 & ITC3 \\
\hline Matière sèche (g / $100 \mathrm{~g} \mathrm{MF)}$ & $32,89 \pm 0,57 a$ & $34,73 \pm 0,65^{b}$ & $34,94 \pm 0,5^{b}$ & $35,73 \pm 1,02^{b}$ \\
\hline Amidon (g / $100 \mathrm{~g} \mathrm{MS})$ & $60,99 \pm 0,07 c$ & $57,61 \pm 0,03^{a}$ & $57,58 \pm 0,01^{a}$ & $57,51 \pm 0,05^{a}$ \\
\hline $\begin{array}{l}\text { Sucres réducteurs (g / } 100 \mathrm{~g} \\
\text { MS) }\end{array}$ & $6,02 \pm 0,13^{a}$ & $12,65 \pm 0,42^{b}$ & $12,37 \pm 0,18^{b}$ & $12,29 \pm 0,31^{b}$ \\
\hline Protéine ( $\mathrm{g} / 100 \mathrm{~g} \mathrm{MS})$ & $0,89 \pm 0,04^{a}$ & $0,88 \pm 0,01^{a}$ & $0,88 \pm 0,04^{a}$ & $0,88 \pm 0,02^{a}$ \\
\hline Fibres (g/ $100 \mathrm{~g} \mathrm{MS})$ & $0,53 \pm 0,008^{a}$ & $0,54 \pm 0,04^{a}$ & $0,60 \pm 0,01^{a}$ & $0,67 \pm 0,13^{a}$ \\
\hline Cendre ( $\mathrm{g} / 100 \mathrm{~g} \mathrm{MS})$ & $0,97 \pm 0,00^{a}$ & $0,98 \pm 0,00^{a}$ & $0,97 \pm 0,00^{a}$ & $0,97 \pm 0,01^{a}$ \\
\hline
\end{tabular}

Moyenne \pm écart-type, $n=3$. Les valeurs d'une même ligne affectée de lettres alphabétiques différentes sont significativement différentes au niveau $5 \%$ selon le test de Tukey de l'analyse des variances. ITCO : igname traitée non congelée (témoin) ; ITC1: igname traitée et congelée pendant un mois ; ITC2 : igname traitée et congelée pendant deux mois ; ITC3 : igname traitée et congelée pendant trois mois.

\section{Caractérisation des paramètres organoleptiques des} mets à base d'igname congelée

Foutou d'igname: L'évolution des paramètres sensoriels (la couleur, l'apparence, l'élasticité avec la fourchette, avec les doigts, dans la bouche, l'adhésivité, le gout et l'odeur) du foutou igname issu des tranches congelées est représentée par la Figure 3. II a été observé une augmentation de l'élasticité des foutous issus des tranches d'ignames congelées par rapport au foutou témoin. Les autres paramètres sensoriels des foutous issus des tranches d'ignames congelées (couleur, l'apparence, l'adhésivité, le goût et l'odeur du foutou igname) ont été jugés similaires à celle du témoin par les panelistes. Hormis l'élasticité, les foutous issus des ignames congelées comme le foutou témoin avaient une couleur moyennement blanche, une apparence lisse, une bonne adhérence, un bon goût et une bonne odeur.

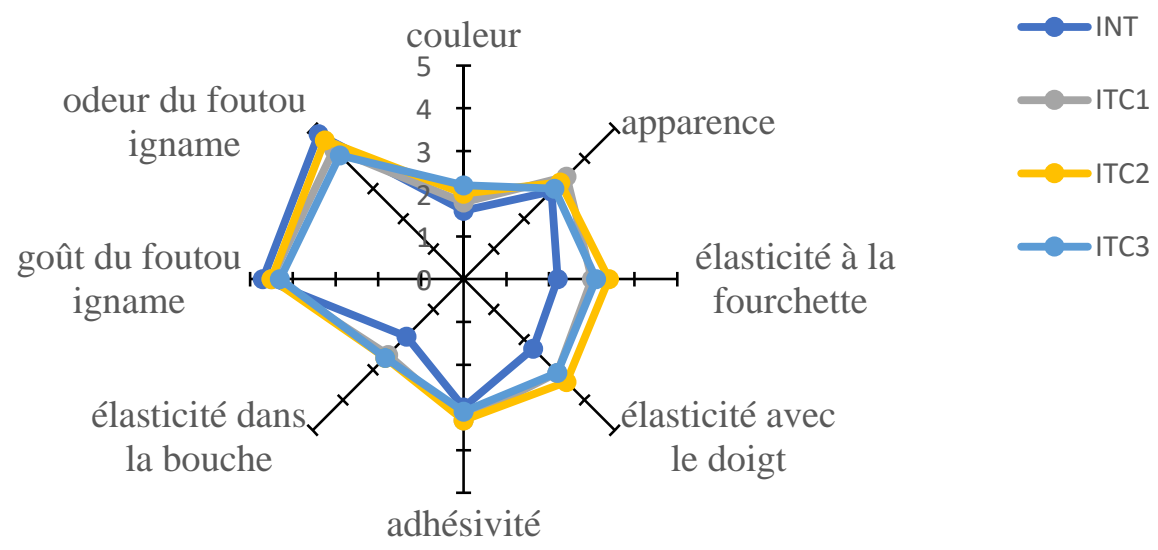

Figure 3 : Profil sensoriel du foutou igname

INT : Igname non traitée ; ITC1 : igname traitée et congelée pendant un mois ; ITC2 : igname traitée et congelée pendant deux mois ; ITC3 : igname traitée et congelée pendant trois mois.

Igname bouillie: L'évolution des caractéristiques organoleptiques des ignames bouillies est représentée par la Figure 4. De façon générale les caractéristiques organoleptiques de l'igname bouillie ont été conservées durant les trois mois de congélation. Les notes obtenues ne montrent aucune différence significative entre les ignames bouillies issues des tranches d'ignames congelées et le témoin pour les paramètres tels que la 
couleur, la fibrosité, la fermeté (avec la fourchette, les doigts et dans la bouche), le goût et l'odeur de l'igame. Les ignames bouillies avaient toutes une couleur moyennement blanche, une fibrosité moyenne, une bonne fermeté, un bon goût et une bonne odeur.

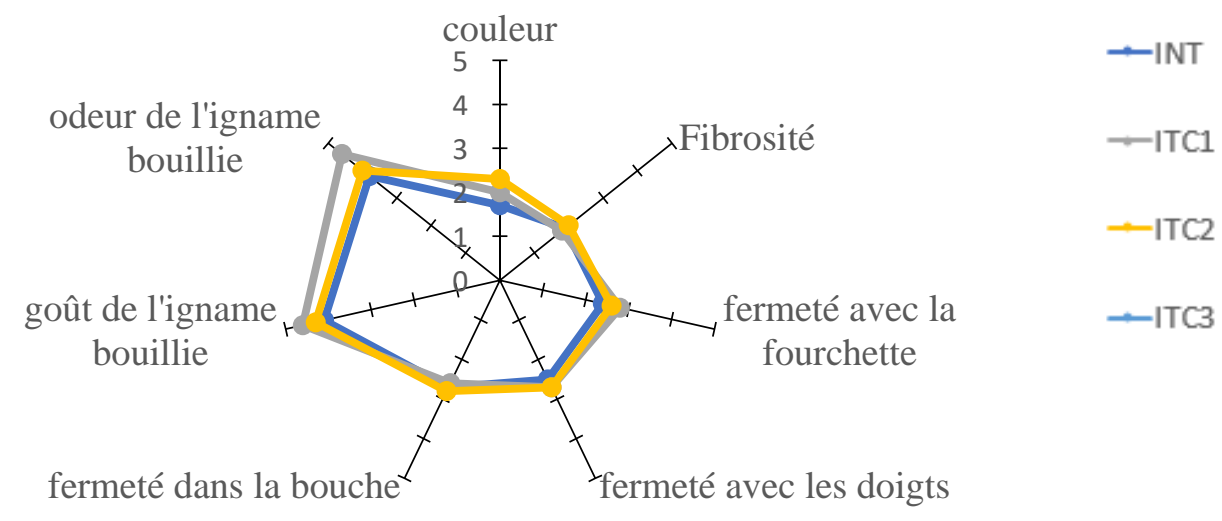

Figure 5 : Profil sensoriel de l'igname bouillie

INT : Igname non traitée ; ITC1 : igname traitée et congelée pendant un mois ; ITC2 : igname traitée et congelée pendant deux mois ; ITC3 : igname traitée et congelée pendant trois mois.

Igname frite : La Figure 5 représente l'évolution des propriétés sensorielles telles que la couleur, la fermeté (avec la fourchette, les doigts et dans la bouche), la croustillance (avec les doigts et dans la bouche), le goût et l'odeur des ignames frites. Une évolution de la fermeté, de la croustillance et de la couleur des frites issues des ignames congelées a été observée au cours du test sensoriel. Les frites devenaient de plus en plus fermes, croustillantes et brunes. Quant au goût et à l'odeur des frites issues des tranches congelées, l'analyse statistique a révélé qu'il n'existe aucune différence significative entre elles et celles de l'igname témoin.

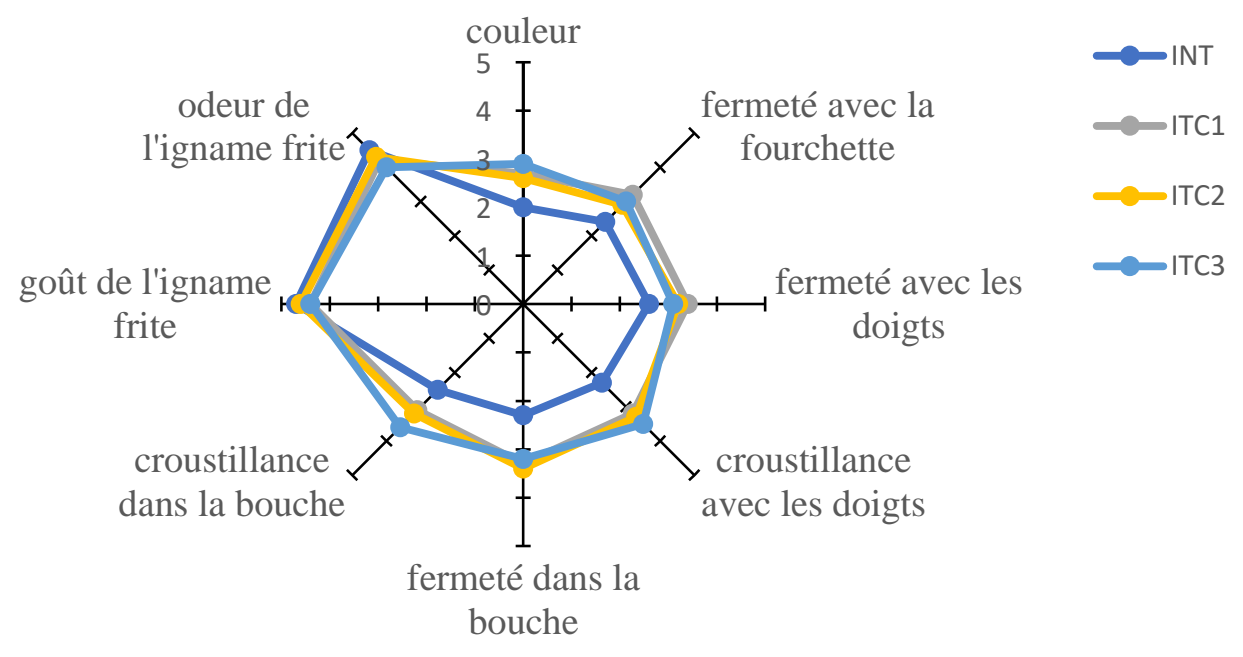

Figure 5 : Profil sensoriel de l'igname frite

INT : Igname non traitée ; ITC1 : igname traitée et congelée pendant un mois ; ITC2 : igname traitée et congelée pendant deux mois ; ITC3 : igname traitée et congelée pendant trois mois. 


\section{DISCUSSION}

L'augmentation de la matière sèche des tranches d'ignames traitées après un mois de congélation pourrait être due à une migration d'eau de l'espace intracellulaire vers la membrane extracellulaire des ignames lors de la congélation. Kowalska et al. (2008) ont observé une perte d'eau plus importante et une augmentation de la matière sèche, après prétraitement de la citrouille par blanchiment à $80^{\circ} \mathrm{C}$ pendant une minute et congelé à $18^{\circ} \mathrm{C}$ pendant 16 heures. En effet, lorsque les vitesses de congélation sont lentes, les premiers cristaux de glace se formant dans le liquide extracellulaire, moins concentré que le milieu intracellulaire entraînent un gradient de pression osmotique permettant la diffusion de l'eau à travers la membrane cellulaire de l'intérieur vers l'extérieur de la cellule (Genot, 2000). Quant à la baisse de la teneur en amidon observé après 1 mois de congélation, elle pourrait s'expliquer par la rupture des parois cellulaires occasionnées par la contrainte exercée sur les granules d'amidon pendant la formation des cristaux de glace (Tao, Wang, Wu, Jin, \& Xu, 2016). L'eau de congélation a exercé une pression sur les granules en raison de la transformation de phase, les cristaux de glace internes ou externes de granules ont occupé plus d'espace qu'une quantité égale de l'eau (Tao et al., 2016). Certains auteurs tels que Waigh et al., (1998) et Perry et Donald (2000) ont signalé, certains désordres structuraux des granules d'amidon qui se produisent à des températures inférieures à zéro lors de la congélation de l'eau dans la fécule de pomme de terre. Mareček et al., (2013) ont également observé une diminution de plus de $14 \%$ de la teneur d'amidon après 6 mois de congélation de différentes variétés de pommes de terre. D'après Singh et Kaur (2009) les cristaux de glace imposent une contrainte de cisaillement sur les granules d'amidon gonflés, les contractants et les perturbants. L'amylopectine concentrée est sujette à l'agrégation moléculaire, ce qui entraîne le rétrécissement des granules d'amidon. Inversement, l'augmentation de la teneur en sucre réducteur est due à la dégradation de l'amidon lors de la congélation. Raigond et al. (2015) ont observé une augmentation de $9,13 \%$ des sucres réducteurs contenus dans les frites de pommes de terre après 180 jours de

\section{CONCLUSION ET APPLICATION DES RÉSULTATS}

Au terme de cette étude, il conviendrait de dire que la congélation a eu une légère influence positive sur certains paramètres physico-chimiques et sensoriels des tranches d'ignames. De façon générale, les tranches d'ignames ont été conservées sur une période congélation. En effet, aux températures d'entreposage inférieures à $10^{\circ} \mathrm{C}$, l'ampleur de l'augmentation des sucres réducteurs est d'autant plus importante lorsque la température est basse jusqu'à point de congélation (Singh et Kaur, 2009). En ce qui concerne le test sensoriel, il a été question de suivre l'évolution de différentes caractéristiques organoleptiques des mets issus de tranches d'ignames étudiées. Les résultats ont révélé la conservation des caractéristiques sensorielles au niveau de l'igname bouillie et l'augmentation de l'élasticité au niveau du foutou ainsi que le changement de la couleur, la fermeté et la croustillance des frites. L'augmentation de l'élasticité du foutou est liée aux transformations des propriétés de l'amidon et au ramollissement des tissus provoqués par la formation de cristaux lors de la congélation (Gallego-Castillo \& AyalaAponte, 2018). Ce résultat est similaire à celui d'Alvarez et Canet (1999) qui ont étudié l'effet des ingrédients et de la congélation sur les propriétés rhéologiques de la purée de pommes de terre. Ces auteurs ont observé une amélioration de l'élasticité de la de la pommes de terre en purée sous forme congelée. Ce fait serait dû à la formation de macrocristaux de glace provoquée par un taux de congélation lent qui a affecté l'intégrité de granulés d'amidon. En plus de cela, lors du chauffage, certains matériaux solubles se sont écoulés de la surface grossière et ont permis une plus grande liberté du gonflement des molécules d'amylopectine (Tao et al., 2016). De plus selon (YU, LI, Xu, \& Zhou, 2008), pendant la congélation extracellulaire, la déshydratation et le rétrécissement des cellules peuvent provoquer la rupture ou le pliage des membranes cellulaires. La couleur dorée plus accrue observée après friture des ignames congelées est liée à la teneur élevée des sucres réducteurs dans les ignames. En effet, les sucres réducteurs participent à la réaction de brunissement de Maillard avec les acides aminés libres pendant la friture, ce qui donne des frites de couleur brun foncé (Singh et Kaur, 2009). Les tubercules de pomme de terre stockés à des températures inférieures à 9 et $10^{\circ} \mathrm{C}$ entraînent des concentrations élevées de sucres réducteurs tels que le glucose et le fructose les permettant de donner une coloration brune après friture (Singh et Kaur, 2009).

de 3 mois et ont été apte pour la confection du foutou, des frites et bouillie d'igname. Cependant, malgré la capacité qu'a la congélation de conserver les ignames, il a été observé une légère diminution de la teneur en l'amidon. II serait intéressant d'avoir des unités de 
fabrication d'ignames congelées. Cette initiative permettra non seulement de rendre l'igname disponible tout le long de l'année en contribuant de ce fait à la sécurité alimentaire mais aussi de diminuer les pertes

\section{RÉFÉRENCES}

Alvarez, M. D., \& Canet, W. (1999). Rheological properties of mashed potatoes made from dehydrated flakes : effect of ingredients and freezing. European Food Research and Technology, 209(5), 335-342. doi : 10.1007/s002170050505

Amani, N. G., Buléon, A., Kamenan, A., \& Colonna, P. (2004). Variability in starch physicochemical and functional properties of yam (Dioscorea sp) cultivated in Ivory Coast. Journal of the Science of Food and Agriculture, 84(15), 2085-2096. doi:10.1002/jsfa.1834

AOAC. (1990). Official methods of analysis of the Association of official analytical chemists, 15 th Edition. Washington, DC.

Assiedu, R. (2003). Yams in west Africa : production and collaborative research. (p. 173-176), Communication présentée au Développement durable de la production et de la consommation de l'igname en Côte d'Ivoire, Abidjan, Côte d'Ivoire.

Bakayoko, G. A., Kouamé, K. F., \& Boraud, N. K. M. (2017). Culture de l'igname au Centre-Est de la Côte d'ivoire: contraintes, caractéristiques sociodémographiques et agronomiques. Journal of Applied Biosciences, 110(1), 10701. doi:10.4314/jab.v110i1.1

Bernfeld, P. (1955). Amylase $\alpha$ and $\beta$. Methods in Enzymology, p. 149-158.

Coulibaly, A., Degbeu, K. C., Bekoin, A. P. M., Kouakoua, Y. E., Pereko, K. K. A., Dabonné, S., \& Amani, N. G. (2019). Browning Prevention and Sensory Evaluation of Frozen Yam Slices (Dioscorea cayenensis-rotundata Cv Kponan) of Côte D'Ivoire. Asian Food Science Journal, 1-10. doi:10.9734/afsj/2019/v9i430022

FAO. (2009). État des ressources phytogénétiques pour l'alimentation et l'agriculture. Second rapport national (p. 65).

FAOSTAT. (2018). Cultures: Ignames. Repéré à http://www.fao.org/faostat/fr/\#data/QC

FIRCA. (2010). Répertoire de technologies de conservation et de transformation de l'igname et de la banane plantain. post récoltes et permettre d'augmenter les revenus des agriculteurs cela contribuera à la lutte contre la paupérisation des populations surtout celle en milieu rurale.

Gallego-Castillo, S., \& Ayala-Aponte, A. (2018). Changes in physical properties of sweet potato due to effects of thermal pre-treatments for puree production. DYNA, 85, 135-142. doi:10.15446/dyna.v85n207.72876

Genot, C. (2000). Congélation et qualité de la viande. Repéré à http://sbiproxy.uqac.ca/login?url=http://internati onal.scholarvox.com/book/45001445

International Institute of Tropical Agriculture (IITA). (2020). Yam. IITA. Repéré à http://www.iita.org/cropsnew/dioscorial

Konan, S., Bédié, K. A., \& Boda, F. (2015). An evaluation of the proximate and elemental compositions of some yam tuber parts during storage. African Journal of Agriculture and Food Security, 3(4), 128-135. Repéré à http://internationalscholarsjournals.org/print.ph $p$ ?article=an-evaluation-of-the-proximate

Kouakou, A. M., Zohouri, G. P., Dibi, K. E., N'Zué, B., \& Foua-Bi. (2012). Emergence d'une nouvelle variété d'igname de l'espèce Dioscorea alata L., la C18, en Côte d'Ivoire. Journal of Applied Biosciences, 57, 4151-4158.

Kouame, K. honore, Koffi, K. E., Kouassi, K. I., Kouassi, K. M., Kouassi, A. B., Kouakou, A. M., ... N'guetta, A. S.-P. (2016). Influence de l'âge des explants primaires sur la régénération des vitroplants de deux espèces d'ignames en côte d'ivoire: Dioscorea alata et Dioscorea cayenensis-rotundata (Dioscoreacea). Journal of Applied Biosciences, 107, 10471-10479. doi:10.4314/jab.v107i0.12

Kowalska, H., Lenart, A., \& Leszczyk, D. (2008). The effect of blanching and freezing on osmotic dehydration of pumpkin. Journal of Food Engineering, 30-38. doi:10.1016/j.jfoodeng.2007.09.006

Lowry, O. H., Rosebrough, N. J., Farr, A. L., \& Randall, R. J. (1951). Protein measurement with the Folin phenol reagent. The Journal of Biological Chemistry, 193(1), 265-275.

Mareček, J., Frančáková, H., Bojňanská, T., Fikselová, M., \& Ivanišová, E. (2013). CARBOHYDRATES IN VARIETIES OF STORED POTATOES AND 
INFLUENCE OF STORAGE ON QUALITY OF FRIED PRODUCTS, (1), 10.

Perry, P. A., \& Donald, A. (2000). The effects of low temperatures on starch granule structure. Polymer, 41, 6361-6373. doi : 10.1016/S00323861(99)00813-7

Raigond, P., Singh, B., \& Kaundal, B. (2015). CHANGES IN THE STARCH AND FIBER FRACTIONS OF FRENCH FRIES : RESPONSE OF FREEZING, 11.

Saxena, T. M., Raju, P. S., \& Bawa, A. S. (2014). Evaluation of sensory acceptability and storage stability of frozen carrot based dessert. Journal of Food Science and Technology, 51(6), 1203-1207. doi:10.1007/s13197-011-0605-8

Singh, J., \& Kaur, L. (Dir.). (2009). Advances in potato chemistry and technology (1st ed.). Amsterdam : Elsevier [u.a.].

Sun, D.-W. (2012). Handbook of Frozen Food Processing and Packaging. CRC Press. Repéré http://gen.lib.rus.ec/book/index.php?md5=8e97 9adc0f1dd08d8e14cd7b8f7dc1fd

Tao, H., Wang, P., Wu, F., Jin, Z., \& Xu, X. (2016). Effect of freezing rate on rheological, thermal and structural properties of frozen wheat starch. RSC Advances, 6(100), 97907-97911. doi:10.1039/C6RA11287K

Waigh, T. A., Perry, P., Riekel, C., Gidley, M. J., \& Donald, A. M. (1998). Chiral Side-Chain LiquidCrystalline Polymeric Properties of Starch. Macromolecules, 31(22), 7980-7984. doi:10.1021/ma971859c

YU, X. L., LI, X. B., Xu, X.-L., \& Zhou, G. H. (2008). Coating with sodium alginate and its effects on the functional properties and structure of frozen pork. Journal of Muscle Foods, 19, 333-351. doi:10.1111/j.1745-4573.2008.00113.x 